\title{
PROPOSITION D'UNE CRITÉRIOLOGIE DANS LE CHOIX DES MODES DE STRUCTURATION DES HYPERMÉDIAS
}

\author{
Pierre Fastrez ${ }^{1}$ et Hugues Peeters ${ }^{2}$
}

\section{Introduction}

Comme d'autres contributions à ce numéro de Recherches en communication l'auront montré, les savoirs informels ne sont pas des savoirs informes. L'attribut "informel" renvoie à une idée d'informalité et non d'informité. Alors que l'informe exprime l'idée de quelque chose sans forme (souvent associée à l'idée d'affreux par ailleurs...), l'informel exprime l'idée de quelque chose sans forme précise. C'est la précision de la forme qui est en jeu dans la distinction entre formel et informel. La différence entre savoirs formels et savoirs informels porte donc sur le fait que les premiers seraient des savoirs aux formes précises tandis que les seconds seraient des savoirs aux formes imprécises.

Ceci nous laisse donc avec trois notions susceptibles d'être explorées : le savoir, la forme et la précision comme attribut de la forme. Pour notre part, nous nous centrerons essentiellement sur des

1 Aspirant au Fonds National de la Recherche Scientifique (FNRS) - Belgique, membre du GReMS.

2 Institut de Pédagogie et des Multimédias de l'UCL, membre du GReMS.

Recherches en communication, $\mathrm{n}^{\circ} 16$ (2001). 
questions tournant autour la notion de forme. Mais si les "communicologues" que nous sommes s'intéressent aux formes des savoirs, ce n'est pas d'un point de vue épistémologique (du moins pas prioritairement), mais d'un point de vue communicationnel. Ils ne s'intéressent pas aux formes de constitution des savoirs (point de vue épistémologique) mais aux formes d'énonciation et de transmission de ces savoirs, c'est-à-dire essentiellement aux formes sémiotiques.

Traiter du sujet des mises en formes sémiotiques des savoirs dans le cadre des NTIC, se ramène d'abord et avant tout -même si cela n'épuise pas le sujet- aux questions touchant aux interfaces. Le propos de notre article sera de traiter de l'introduction des formes hypertextuelles dans les interfaces des nouveaux médias. Nous envisagerons également la question de ses retombées dans le cadre de la transmission de savoirs.

Premièrement, nous nous interrogerons sur les implications et les enjeux des spécificités de l'hypertexte en ce qui concerne la structuration des documents et l'appréhension de cette structure par l'utilisateur durant la navigation. Ensuite, nous proposerons un ensemble de pistes pratiques pour concevoir les structures des documents hypertextuels. Nous présenterons quatre modes de structuration opposés deux à deux, et nous mettrons en évidence les critères susceptibles de diriger le choix de l'un ou l'autre de ces modes. Et, enfin, nous conclurons en mettant en doute les espoirs tenus notamment dans le monde de l'enseignement quant au fait que les propriétés sémiotiques de l'hypertexte en feraient un dispositif particulièrement susceptible de permettre à l'apprenant d'appréhender facilement (à la mode des savoirs informels) les structures globales de certains savoirs. Nous soutiendrons que c'est sur d'autres plans que l'hypertexte est susceptible d'apporter quelque chose au champ de l'apprentissage.

\section{Importance de la structure dans les hypermédias : la question de la navigation}

Pourquoi la question de la navigation se pose-t-elle de façon plus importante pour l'hypertexte que pour le livre ou d'autres médias traditionnels ? En fait, l'hypertexte force son utilisateur à maintenir une certaine attention au processus de navigation, et donc à l'organisation formelle du document, à sa structure. Développons. 


\section{Naviguer et s'orienter : une tâche complexe}

La lecture d'un livre comme la consultation d'un hyperdocument sont des activités complexes et exigeantes du point de vue cognitif, dont l'une des difficultés consiste à coordonner les différents types de tâches qu'elles englobent avec des ressources cognitives limitées -pensons aux limites de la mémoire de travail ${ }^{1}$. Cependant, les contraintes qui pèsent sur la manipulation d'un hyperdocument et celles propres à la lecture d'un livre diffèrent quelque peu.

Du point de vue de l'accès direct à l'information tout d'abord, la consultation d'un hyperdocument implique que l'on soit toujours "immergé" dans le document : l'espace limité de la surface de l'écran ne donne jamais accès qu'à un fragment du contenu, sans que le reste soit visible. Le texte traditionnel ne subit pas cette contrainte: le déploiement du journal autorise un survol de larges portions de son contenu en un coup d'œil ; la configuration de l'objet-livre, même si elle n'autorise que la lecture d'une double page à la fois, procure des repères sensori-moteurs et visuo-spatiaux qui renseignent le lecteur sur la place qu'occupe le fragment lu dans l'ensemble de l'ouvrage (ne fût ce que l'épaisseur des pages sous la page de gauche et de droite).

Dans le livre, le repérage intuitif qu'offre l'objet est couplé à la structuration et à des mises en forme micro- et macrotypographiques historiquement stabilisées : agencement en chapitres, index, sommaires et tables des matières, mise en forme des titres indiquant leur importance respective, etc. fournissent tous des indices au lecteur qui lui permettent de se repérer instantanément dans un livre.

L'expérience immersive de l'hypertexte tend à produire des effets de lissage sur la valeur différentielle des morceaux d'information qu'il contient. En ne nous permettant que d'avoir le nez collé sur les fragments d'informations, l'hypertexte procède à une mise à plat dans laquelle il devient parfois difficile de prendre conscience d'une hiérarchie, de distinguer l'essentiel de l'accessoire, sans présumer de la présence d'un projet personnel préalable dans le chef de l'utilisateur.

1 Cf. J.-F. Rouet, J. J. Levonen, A. Dillon et R. J. SPIRo (dir.), Hypertext and cognition, Mahwah, New Jersey, Lawrence Erlbaum Associates Inc., 1996. 
La structuration du contenu du livre lui-même renseigne encore le lecteur : de l'ensemble de nos lectures, nous avons tous et toutes abstrait quelques modèles schématiques propres à des types de textes (van Dijk \& Kintsch ${ }^{1}$ parlent de structures superschématiques). Le schéma narratif et le schéma argumentatif sont deux exemples généraux de ces organisations types qui nous permettent d'anticiper d'entrée de jeu la structure du discours inclus dans un ouvrage. Plusieurs études ${ }^{2}$ ont ainsi mis en avant la capacité de lecteurs de revues scientifiques à deviner de façon fiable la position d'un paragraphe isolé au sein de l'article original dont il était issu (introduction, discussion des résultats, etc.).

A contrario, à l'heure actuelle, les hypermédias pèchent par leur défaut d'indices sensori-moteurs et visuo-spatiaux, ainsi que de conventions typographiques et de structuration. Ceci contraint l'utilisateur à mobiliser plus de ressources (que dans le cas du livre) pour gérer activement les tâches navigationnelles propres à sa consultation (c'est-à-dire pouvoir situer l'information consultée dans l'organisation d'ensemble du document, localiser une information recherchée dans cette organisation, trouver le parcours menant à cette information, etc.), là où le dispositif livresque permet au lecteur de se centrer sur le contenu et les tâches dites informationnelles ${ }^{3}$.

C'est l'absence de parcours de lecture par défaut unique de l'hypertexte qui force les choix de navigation dans le chef de l'utilisateur, là où le lecteur de livres peut en quelque sorte "se reposer" sur ce que le livre lui propose. La navigation mobilise donc plus l'utilisateur d'hypermédias que le lecteur de livres : la difficulté de percevoir la structure formelle du document, qui est spécifique au support, force

1 T. A. VAN DIJK \& W. KINTSCH, Strategies of discourse comprehension, New York, Academic Press, 1983.

2 Résumées dans A. Dillon, C. MCKNight \& J. Richardson, " Space - The final chapter or Why physical representations are not semantic intentions", in C. MCKNight, A. Dillon \& J. Richardson (dir.), Hypertext. A psychological perspective, Chichester, Ellis Horwood Ltd, 1993, pp. 178-179.

3 Tâches informationnelles et navigationnelles correspondent à ce que la littérature identifie (implicitement ou explicitement) comme étant les deux types principaux de tâches de l'utilisateur d'hyperdocuments (Cf. H. KIM et S. C HIRTLE, "Spatial metaphors and disorientation in hypertext browsing ", Behaviour \& Information Technology, vol. 14, $\mathrm{n}^{\circ} 4,1995$, pp. 239-250; M. THÜRING, J. HANNEMANN et J.M. HAAKE, "Hypermedia and cognition, designing for comprehension", Communications of the $A C M$, vol. $38, \mathrm{n}^{\circ} 8$, août 1995 , pp. 57-66). Il est bien entendu que ces deux types de tâches empiètent l'un sur l'autre, et qu'il est peu raisonnable de les étudier chacun en vase clos. 
une attention plus grande à cette structure. Plusieurs recherches ont ainsi mis en avant le lien étroit entre la capacité de l'utilisateur à percevoir cette structure et la facilité avec laquelle il navigue dans le document. Ainsi, Dillon, dans un article consacré à l'étude des différences interindividuelles dans l'utilisation des hypermédias, affirme :

There exists one such difference that seems central to ensuring the navigability, and hence, the usability of digital environments -the user's ability to perceive structure or shape in information space'.

\section{Hypertexte et associativité : un parallèle prudent}

La difficulté que représente la navigation pour l'utilisateur d'hypermédias -qui se manifeste notamment par les problèmes de désorientation ou de surcharge cognitive ${ }^{2}-$ a suscité de nombreuses recherches consacrées à la question des outils de navigation, que la recherche sur les hypertextes des premiers temps avait négligé. L'analogie entre les possibilités d'association du support hypertextuel et le fonctionnement associatif de l'esprit humain a en effet nourri chez certains l'espoir qu'un tel support permette un apprentissage totalement transparent.

Le découpage de l'information en nœuds connectés par des liens, propre à l'hypertexte, offre la possibilité de concevoir des documents créant un réseau d'associations libres entre informations, au gré de leur proximité sémantique (ou de tout autre critère). Du parallèle entre ce principe de structuration et l'associativité propre à l'esprit humain, certains se sont mis à penser qu'il " suffisait" de structurer les informations dans un hypertexte "comme dans l'esprit d'un expert" pour que l'apprenant navigue directement dans les connaissances de

1 A. Dillon, "Spatial-Semantics. How users derive shape from information space", Journal of the American Society for Information Science, vol. 51, $\mathrm{n}^{\circ} 6,2000$, p. 522.

2 Cf. H. Kim et S. C. HirtLe, op. cit.; J.-F. Rouet et J. J. Levonen, "Studying and learning with hypertext. Empirical studies and their implications ", in J.-F. ROUET, J. J. LEVONEN, A. Dillon et R. J. SPIRo (dir.), Hypertext and cognition, op. cit. pp. 9-23; M. Thüring, J. Hannemann et J. M. HaAKe, op. cit. ; A. Tricot et L. ChANQuoy, "Introduction: la charge mentale, "vertu dormitive" ou concept opérationnel ?", in A. TRICOT et L. CHANQUOY (dir.), La charge mentale, Psychologie Française, ${ }^{\circ}$ 41, vol. 4, Paris, Dunod, 1996, pp. 313-318. 
l'expert, et apprenne le plus naturellement du monde ${ }^{1}$. La suite devait leur donner tort...

Cette thèse repose sur la conception selon laquelle l'esprit humain fonctionne essentiellement par association : la mémoire de l'être humain serait faite d'un vaste réseau d'informations reliées entre elles de multiples façons. Pour les tenants de cette thèse, "knowledge is represented cognitively in some form of semantic network or web" 2 . Cette idée est soutenue par plusieurs concepts forgés par la psychologie cognitive, tels ceux de réseau sémantique ou de schéma.

David Jonassen est sans doute le meilleur représentant de la position exposée ici. Jonassen a mis sur pied plusieurs expériences reposant sur des hyperdocuments d'apprentissage dont la structure avait été élaborée par "projection" de la structure sémantique des connaissances d'un expert du domaine (nous ne discuterons pas ici des méthodes de "projection " employées dans le cadre de la conception de ces documents). Pourtant, aucun des résultats obtenus ne permet d'affirmer de façon ferme qu'un tel mode de structuration facilite l'acquisition de connaissances, ni (c'est plus spécifiquement ce sur quoi se penche Jonassen) la mâtrise de la structure de ces connaissances.

So, merely showing learners structural relationships, without a purpose for doing so, is probably not sufficient to result in meaningful encoding of that information ${ }^{3}$.

A posteriori, il apparaît que l'échec de ce courant de recherche repose sur deux éléments. D'une part, le parallèle entre spécificités sémiotiques du support et spécificités cognitives de l'humain a fait croire à tort à une transparence totale du passage de l'un à l'autre.

1 Cf. A. TRICOT et C. BASTIEN, "La conception d'hypermédias pour l'apprentissage. Structurer des connaissances rationnellement ou fonctionnellement ?", in E. BRUILlARD, J.-M. BALDNER et G.-L. BARON (dir.), Hypermédias et apprentissages. Préactes du colloque, (CREPS de Chatenay-Malabry, 9-11 mai 1996), ronéotées, 1996, pp. 23-33.

2 A. DILLON, "Myths, misconceptions, and an alternative perspective on information usage and the electronic medium", in J.-F. ROUET, J. J. LEVONEN, A. DILLON et R. J. SPIRO (dir.), Hypertext and cognition, op. cit., p. 28.

3 D. H. JONASSEN, "Effects of semantically structured hypertext knowledge bases on user's knowledge structures", in C. MCKNIGHT, A. DILLON \& J. RICHARDSON (dir.), Hypertext. A psychological perspective, op. cit., p. 164. 
Dillon et alii ${ }^{1}$ apportent un argument intéressant dans ce cadre, en faisant une distinction nette entre "espace sémantique" et "espace du document" : on ne navigue jamais directement dans les connaissances du concepteur, dans son argumentation, dans ses intentions, disent-ils. On navigue dans une représentation physique de celles-ci, qui ne peut leur être totalement équivalente. Le parallèle entre les deux navigations n'est valable qu'au prix de cette distinction. Il n'est donc pas suffisant d'offrir à l'utilisateur un document réifiant la structure des connaissances d'un expert pour que celui-ci apprenne. Il faut également lui fournir les outils ${ }^{2}$ lui permettant de se réapproprier cette structure, de s'y repérer, de la faire sienne.

D'autre part, il y a confusion manifeste entre deux états des connaissances diffusées : d'un côté les connaissances en construction de l'apprenant, issues d'un processus d'élaboration progressive, et de l'autre les connaissances fermement établies et structurées de l'expert. En voulant faire naviguer le néophyte "directement dans les connaissances de l'expert", on a fait l'impasse sur le processus de construction de ces connaissances, en pensant que le néophyte serait à même d'assimiler une structure finalisée plutôt que de construire et de structurer lui même progressivement son propre domaine de connaissances.

\section{Comment structurer ? \\ Alternatives dans la structuration imprimée à un hypermédia}

De la première partie de notre exposé, on tire deux nécessités pour toute conception d'hypertexte :

- la nécessité de structurer, de réfléchir à la façon dont on organise le document que l'on conçoit ;

- la nécessité de donner des outils de navigation permettant d'appréhender cette structure et de s'en servir dans des tâches liées au contenu du document (la navigation est rarement une fin en soi).

1 A. Dillon, C. MCKnight \& J. Richardson, "Space - The final chapter...", op. cit.

2 Index, plans, menus, boutons, mise en écran, bref c'est tout l'interface qui est concerné ici. 
La suite de notre article dégagera deux axes susceptibles de régir le mode de structuration adopté. Nous articulerons les pôles de ces deux-ci à un ensemble de critères permettant d'opérer un choix à leur égard dans la construction des hypertextes.

\section{Structure rationnelle vs structure fonctionnelle}

Cette première distinction a été initialement opérée par André Tricot et Claude Bastien ${ }^{1}$. Le concept de structuration fonctionnelle renvoie pour eux à la façon dont les connaissances sont organisées dans la mémoire telles que mobilisées par l'individu en contexte. Travaillant à partir des études de Le Ny sur le rôle du contexte dans la mobilisation des connaissances, Tricot et Bastien se focalisent sur les connaissances orientées vers la réalisation d'un but, d'un objectif. Selon eux, un même individu mobilise différemment les mêmes concepts en fonction du contexte (et plus spécifiquement de l'objectif à atteindre) dans lequel il les utilise ${ }^{2}$. Et pour eux :

La mobilisation de connaissances fonctionnelles dans deux contextes différents n'entraîne pas simplement une variation dans la hiérarchisation des attributs, mais bien la mobilisation de fonctionnalités différentes, de liens différents, et de réseaux différents : un même concept peut être localisé à deux endroits différents ${ }^{3}$.

Les connaissances fonctionnelles concernant un même concept diffèrent donc en fonction du contexte, ainsi que du sujet qui les mobilise. Devant cette variabilité, comment concevoir un hypermédia dont la structure suivrait une infinité de structurations possibles, en fonction d'une infinité de contextes possibles ? Qui plus est, la "fonctionnalisation" des connaissances (la capacité de les utiliser dans des contextes particuliers multiples) ne peut avoir lieu dans le premier temps de l'apprentissage :

1 A. Tricot et C. Bastien, op. cit.

2 Les auteurs citent l'expérience de Le Ny concernant les traits sémantiques convoqués en mémoire par les sujets à l'écoute du mot " piano " dans deux phrases (c.-à-d. deux contextes) différent(e)s : "Lydia jouait délicieusement une sonate sur son piano " et "Les déménageurs ont eu de la peine à apporter le piano ". Le piano de la sonate n'est pas celui des déménageurs : il correspond à deux types de connaissances fonctionnelles différentes.

3 A. TRicot et C. BASTIEN, op. cit., p. 26. 
Car l'établissement d'un lien fonctionnel entre deux connaissances est un objectif d'apprentissage à beaucoup plus long terme que la simple consultation d'un hypermédia!

Le principe que nous défendons est que plutôt que d'imiter les connaissances du sujet, le concepteur doit, par une structure claire, rationnelle, relativement indépendante du contenu, permettre au sujet de localiser des informations et de comprendre les liens qu'il y a entre elles dans le système ${ }^{2}$.

Pour Tricot et Bastien, un " bon " hypermédia d'apprentissage est donc structuré rationnellement. Il offre une structure d'ensemble logique, partiellement indépendante du contenu du document ${ }^{3}$, et surtout indépendante du contexte dans lequel seront utilisées les connaissances. Les connaissances organisées rationnellement sont donc autonomes, pour mieux pouvoir être fonctionnalisées à long terme. Elles font l'objet d'une segmentation et d'un classement logique. Par exemple, si l'on conçoit une structure rationnelle comme hiérarchique, chaque information appartiendra à une section, une sous-section, etc., la définition des sections s'élaborant sur base de regroupements logiques, essentialistes, des informations.

Selon nous, il convient néanmoins de recadrer le parti pris par Tricot et Bastien, en fonction du type de tâche qui sous-tend la consultation de l'hypermédia conçu, dans le cadre de laquelle les informations contenues dans l'hypermédia sont utilisées. C'est en fonction de la délimitation de la tâche que la structuration de l'hyperdocument doit se calquer soit sur la structure de l'objet, des informations contenues (structuration substantialiste, rationnelle), soit sur la structure de la tâche elle-même (structuration fonctionnelle).

Le point de vue de Tricot et Bastien est parfaitement tenable tant que l'hyperdocument est conçu pour être consulté dans le cadre d'une tâche "ouverte", qui ne se termine pas avec la consultation. Nous appellerons ce type de consultation " transitive". Dans une telle situation d'apprentissage, les connaissances acquises n'ont pas fini de

1 Ibid., p. 31.

2 Ibid., p. 23.

${ }^{3} \mathrm{La}$ " charpente" de base ne prend pas en compte toutes les associations sémantiques propres aux informations concernées. Si l'on distingue les liens organisationnels (propres à cette "charpente", au découpage en sections, etc.) des liens associatifs ou transversaux, les associations sémantiques ne sont prises en charge que par les seconds. 
servir après consultation, et doivent pouvoir être mobilisées par après dans une multiplicité de contextes. La structuration rationnelle est donc la plus appropriée ${ }^{1}$.

Aux consultations transitives (ou plutôt aux connaissances transitives, structurées rationnellement) s'opposent les consultations intransitives. Dans ce cas de figure, l'hyperdocument et les informations qu'il contient sont conçus pour permettre à l'utilisateur de mener à bien une tâche délimitée, qui est soit incluse à la consultation ellemême $^{2}$, soit concomitante à celle-ci ${ }^{3}$. Dans un tel cadre, la structuration fonctionnelle semble la plus appropriée. Nous avons en effet montré plus haut qu'adopter une structuration fonctionnelle équivaut à présenter les informations à l'utilisateur dans l'ordre et l'organisation propres à leur utilisation en contexte. Hors quand le contexte d'utilisation est la consultation, la structuration fonctionnelle du document

1 Il peut paraître de prime abord surprenant de qualifier les connaissances qui procèdent de telles situations d'apprentissage de "transitives", alors qu'on a coutume de se représenter les connaissances savantes comme des matières comportant un degré élevé d'autonomie. Mais le point de vue que nous développons ici suppose comme postulat qu'il n'existe pas de savoirs ou de connaissances totalement désintéressé(e)s. Tout apprentissage, aussi conceptuel et abstrait soit-il, a toujours, d'une manière ou d'une autre une visée pratique. Le choix d'une structuration rationnelle, abstrayant les connaissances concernées d'un contexte d'utilisation particulier, a moins pour ambition de susciter un savoir abstrait de tout contexte, qu'une connaissance applicable à une infinité de circonstances.

Ainsi, la Science, ce bastion de l'autosuffisance, détaché de toute contingence, n'est pas à identifier trop rapidement comme parangon de l'intransitivité. Le projet de la science a en effet définitivement cessé depuis le début de la modernité d'être un projet gratuit et désintéressé. Chez Bacon comme Descartes, l'idéal contemplatif des anciens n'est plus une fin en soi, mais un moyen à l'homme pour servir à son projet d'émancipation. Dans le Novum Organum, le monde n'est plus à considérer comme ensemble d'essences à comprendre et méritant le respect méditatif, mais comme quelque chose à disséquer et réduire à un enchaînement mécanique de causes, et ce pour en devenir " maître et possesseur " ("Scientia proper potentiam "). Bref, le projet de la science moderne est donc bien, à travers la construction d'énoncés très abstraits et autosuffisants, de développer des procédés les plus transitifs possibles, car applicables au plus grand nombre de situations envisageables. La science correspond en fait depuis la modernité au comble de la transitivité.

2 Rechercher un programme de formation et $s^{\prime} y$ inscrire ; acheter un livre sur amazon.com : c'est en naviguant que je m'inscris / j'achète.

3 C'est-à-dire que le temps de la consultation est le temps de réalisation de la tâche. Suivre une leçon du tutoriel d'un logiciel de mise en page ; résoudre un problème informatique grâce à l'aide en ligne du système d'exploitation (MacOS, Windows) : l'aide propose sur support hypertextuel des informations permettant de résoudre pas à pas le problème. 
doit suivre celle de la tâche à réaliser. Plutôt que de structurer l'hyperdocument en fonction d'un classement logique propre à l'information, on a donc tout avantage à partir de la structuration de la tâche pour structurer le document conçu.

Concrètement, il s'agit d'organiser les nœuds en fonction des besoins de l'utilisateur dans le courant de la réalisation de la tâche : à chaque étape, les liens proposés correspondent aux choix alternatifs auxquels l'utilisateur est confronté pour mener sa tâche à bien, aux différentes ressources dont il peut avoir besoin à ce stade, etc. Dans le domaine des hyperdocuments éducatifs, ce mode de structuration trouverait application dans l'ensemble des documents proposant un apprentissage par l'action, la manipulation, l'expérimentation et la simulation (expériences scientifiques, etc.).

La structuration fonctionnelle des hypermédias calque donc ici la structure du support sur celle de la tâche à réaliser par l'utilisateur. Ceci comporte deux implications : d'une part, il revient au concepteur de définir cette tâche et sa structure explicitement, d'autre part, cette explicitation conduit à une standardisation de ladite tâche. Dans la mesure où il part de l'activité de l'utilisateur pour définir la structure de l'hyperdocument, le concepteur définit les actions possibles et impossibles dans celui-ci, et décide de la façon dont l'utilisateur remplira sa tâche. Il limite par là l'autonomie de celui-ci.

Structurer fonctionnellement, c'est donc " figer" partiellement le flux d'activité de l'utilisateur, au risque que la structuration adoptée ne corresponde pas à ce que celui-ci attend.

Si la structuration fonctionnelle est appropriée lorsqu'il s'agit de résoudre un problème limité, elle compromet l'ambition de susciter un apprentissage "transférable " à d'autres situations, puisque les informations proposées répondent à une tâche circonscrite, au contraire de la structuration rationnelle.

\section{Simplicité et " utilisabilité " vs complexité intentionnelle}

Nous l'avons vu plus haut, la navigation et le "poids " qu'elle représente pour l'utilisateur constitue une préoccupation de la recherche sur les hypermédias. De nombreux chercheurs et concepteurs se sont ainsi penchés sur le probléme de la désorientation. L'orientation et la navigation représente a priori une difficulté pour l'utilisateur d'hypermédias. Leur travaux ont pour objectif d'évaluer 
par quels moyens et dans quelle mesure on pouvait alléger le fardeau de l'utilisateur.

Mais en fait, deux points de vue opposés sont soutenables devant les exigences que pose la navigation à l'utilisateur. Le premier mise sur une structuration la plus simple et intelligible possible, tandis que le second tente de tirer parti de la complexité de la structure pour initier l'utilisateur à la complexité du savoir diffusé.

\section{La simplicité avant tout et le concept d'usability}

Du constat de base qu'il existe un problème de désorientation dans les hypertextes, différents auteurs ont proposé des solutions variées, fondées soit sur l'entraînement et la familiarisation de l'utilisateur au dispositif ${ }^{1}$, soit sur la cohérence du contenu' ${ }^{2}$, soit encore sur un ensemble d'outils de navigation ou de métaphores rendant la structure du document explicite et intelligible ${ }^{3}$. Ce dernier type de travail suppose que la structure soit la plus simple à comprendre possible, afin de rendre la tâche la plus facile possible à l'utilisateur.

La structure du document, ainsi que ses parcours au sein de celleci, doivent donc être intelligibles et facilement reconstituables. Au final, l'utilisateur doit pouvoir se centrer un maximum sur les tâches "informationnelles" (propres au contenu) sans avoir à faire d'effort particulier en ce qui concerne les tâches navigationnelles.

Ultimately, users visit your website for its content. Everything else is just the backdrop. The design is there to allow people access to the content. ${ }^{4}$

L'auteur de cette citation (Jakob Nielsen) est l'un des représentant les plus en vue dans le monde du webdesign d'un courant de recherche centré sur le concept d'usability ("utilisabilité " en

1 J.-F. ROUET et J. J. LEVONEN, op. cit.

2 M. ThÜring, J. HANNEMANN et J. M. HAAKE, op. cit.

3 A. DILlon, C. MCKNIGHT et J. RICHARDSON, "Navigation in hypertext : a critical review of the concept ", in D. DIAPER, G. COCKTON, D. GILMORE et B. SHACKEL (dir.), Human-Computer Interaction - INTERACT' 90 . Proceedings of the IFIP TC 13 Third International Conference, (Cambridge, U.K., 27-31 août 1990), Amsterdam - New York, North Holland, 1990, pp. 587-592; H. KIM \& S. C. HIRTLE, op. cit.

4 J. NIELSEN, Designing web usability. The practice of simplicity, Indianapolis, Indiana, New Riders Publishing, 1999, p. 99. 
français). Selon lui, l'enjeu principal dans la conception d'un hyperdocument est de le rendre utilisable par les utilisateurs. Tout le projet de conception doit partir des besoins de ce dernier, de façon à concevoir un document dans lequel il puisse naviguer et y trouver les informations qu'il cherche sans aucune difficulté. L'ensemble du design doit donc être au service de l'utilisateur.

Le user-centered design se situe au croisement de plusieurs disciplines, au nombre desquelles on compte l'ergonomie, les études en interaction homme-machine, la psychologie et l'ingénierie industrielle. Il ne se limite en fait pas à la conception d'hyperdocuments, mais se centre sur le design des artefacts informationnels en général. Le point de vue adopté par le usercentered design est synthétisé par Donald Norman (l'un des pères de ce courant) comme suit :

Design should :

- Make it easy to determine what actions are possible at any moment (make use of constraints).

- Make things visible, including the conceptual model of the system, the alternative actions, and the results of actions.

- Make it easy to evaluate the current state of the system.

- Follow natural mappings between intentions and the required actions; between actions and the resulting effect; and between the information that is visible and the interpretation of the system state ${ }^{1}$.

En bref, l'utilisateur doit être capable à tout moment de déterminer ce qu'il peut faire ou ce qu'il doit faire, et ce qui se passe dans le système. Le modèle conceptuel sous-jacent à l'artefact ou au document (c'est-à-dire le modèle mental permettant d'expliquer la façon dont il fonctionne) doit être intelligible à partir de l'artéfact lui-même, rendant prévisibles les effets des actions de l'utilisateur sur le système. Les actions possibles, leurs résultats et l'état actuel du système doivent être visibles, et leur expression ou les contrôles y correspondant doivent dans la mesure du possible exploiter des projections (mappings) naturels : la relation unissant les deux éléments (le contrôle et l'action, par exemple) est dans ce cas non conventionnelle (analogique, de l'ordre de l'icône ou de l'indice, dirait le communicologue). L'exemple le plus simple est celui du

1 D. NoRman, The Design of Everyday Things, New York, Basic Books, 1988, p. 188. 
volant d'automobile : le sens de rotation correspond au sens dans lequel la voiture tourne (dans la mesure où le mouvement opéré par le conducteur part du dessus du volant).

Le user-centered design impose que le temps d'apprentissage nécessaire à la manipulation correcte du document soit minimisé : ce n'est pas à l'utilisateur à essayer de comprendre la logique de votre site Web ou de votre CD-ROM, c'est à vous de le concevoir de façon intuitive pour lui, de sorte qu'il puisse l'utiliser d'emblée. La manipulation du dispositif doit pouvoir s'apprendre sans instruction extérieure.

Cet ensemble de consignes et de contraintes implique que le processus de conception et de structuration d'un hyperdocument parte non pas des informations à mettre à disposition, et de la façon dont on voudrait les présenter, mais de ce dont l'utilisateur a besoin, de ce pourquoi il viendra consulter le document. Au niveau plus spécifique de la structuration de l'hyperdocument, il s'agit de simplifier au maximum ladite structure, ainsi que la façon de la présenter à l'écran.

Étant donné le cadre éducatif dans lequel nous nous plaçons ici, nous pouvons nous permettre de prendre quelque peu distance vis-àvis de cette logique, ce que nous allons tenter de faire en présentant une position alternative à celle développée jusqu'ici.

\section{La Cognitive Flexibility Theory (CFT)}

La CFT est une théorie de l'apprentissage qui se centre sur l'acquisition de connaissances complexes appartenant à des domaines mal structurés (ill-structured domains) ${ }^{1}$. Pour enseigner de telles matières, la CFT prône de passer non par leur simplification, leur découpage en unités simples, etc. mais justement par le maintien de leur complexité d'emblée (dans une mesure gérable par l'apprenant), la mise en évidence des liens multiples entre notions, entre concepts abstraits et cas d'application multiples, etc. Les tenants de cette théorie détaillent cinq antidotes contre la sur-simplification nuisible à

1 R. J. Spiro, P. J. Feltovich, M. L. Jacobson et R. L. Coulson, “ Cognitive Flexibility, Constructivism and Hypertext. Random access instruction for advanced knowledge acquisition in ill-structured domains ", Educational Technology, $\mathrm{n}^{\circ} 31$, mai 1991, pp. 24-33. 
l'apprentissage (et notamment au développement de la capacité à appliquer les connaissances acquises à de multiples contextes) ${ }^{1}$ :

1. Recourir à des représentations conceptuelles multiples des connaissances ;

2. Relier les concepts abstraits à différents exemples applicatifs ;

3. Introduire d'emblée la complexité du domaine (... au lieu d'isoler des unités conceptuelles limitées avant de les assembler);

4. Mettre l'accent sur la nature réticulaire et intégrée des connaissances ;

5. Encourager l'assemblage des connaissances conceptuelles abstraites et exemplatives, dans le cadre d'une application donnée ou d'une tâche de résolution de problème.

Selon les tenants de la CFT, l'hypertexte constitue un outil pédagogique permettant de mettre en ouvre ces antidotes.

Dans un de leurs articles, Jacobson et Spiro comparent à travers une expérience les effets d'un support hypertextuel concernant une matière mal structurée et complexe (les relations technologie société) et appliquant ces antidotes à un apprentissage par drill (questions réponses successives) sur le même sujet. Comparé au groupe de sujets subissant le drill, le groupe de sujets étudiant avec l'hypertexte fait de moins bons scores aux tests de mémoire mais de meilleurs scores aux tests impliquant le transfert des connaissances acquises à de multiples situations.

Overall, these findings suggest-hypertext learning environments that present the instructed knowledge by explicitly demonstrating critical interrelationships between abstract and case-specific knowledge components in multiple contexts will help prepare students to use knowledge in new ways and in new situations ${ }^{2}$.

Les potentialités hypertextuelles -telles qu'exploitées à travers les cinq principes de la CFT énoncés plus haut- apparaissent donc comme un adjuvant à l'acquisition de connaissances complexes et mal structurées.

1 M. J. JACOBSON et R. J. SPIRo, "Hypertext learning environments, cognitive flexibility, and the transfer of complex knowledge. An empirical investigation", Journal of Educational Computing Research, vol. 12, n 4, 1995, pp. 301-333.

2 Ibid., p. 301. 


\section{Implication sur la structuration hypertextuelle : un changement de priorité}

A partir des thèses de la CFT sur la conception des hyperdocuments, on peut alors fonder dans le contexte de l'éducation du moins un point de vue qui prend le contre-pied de celui de l'" utilisabilité ".

Comme les travaux de Dillon évoqués plus haut le montrent, la capacité à percevoir la structure d'un hypertexte rend la navigation de celui-ci beaucoup plus facile ${ }^{1}$. Cette capacité n'est pas quelque chose qui va de soi dans les hypertextes. Ceci a amené la plupart des concepteurs d'hypermédias à penser qu'il fallait faciliter un maximum la tâche des utilisateurs, d'une part en concevant des structures les plus simples et les plus intelligibles possibles, d'autre part en les rendant les plus manifestes possibles dans l'interface (mise en écran, liens, etc.). C'est le point de vue des défenseurs de l'usability.

Nous voudrions montrer, qu'en prolongeant le point de vue de la CFT, le parti inverse peut également se justifier. L'associativité propre à l'hypertexte est un adjuvant exploitable dans le cadre de l'apprentissage de matières complexes et mal (ou pas) structurées. L'information est alors présentée dans des contextes multiples, reliée à des exemples variés, expliquée depuis différentes perspectives, etc. Les notions exposées le sont en inter-relation les unes avec les autres, les liens entre elles étant ce sur quoi on met l'accent ${ }^{2}$.

Concevoir une structure complexe, présentant chaque information dans des parcours de lecture (et d'interprétation) multiples, et forcer l'utilisateur à faire l'effort de comprendre cette structure (inscrite dans le support), de s'arrêter pour s'interroger sur celle-ci, peut également constituer une démarche productive dans le cadre d'un

1 Cf. A. Dillon, "Spatial-Semantics...", op. cit.

2 C'est précisément la démarche qui a guidé la conception de l'hyperdocument "Technology and the twentieth century : impact on society and culture ", conçu dans le cadre de l'expérimentation évoquée au point précédent (cf. M. J. JACOBSON et R. J. SpIRo, op. cit.). Celui-ci croise six cas de technologies propres à nos sociétés avec six thématiques d'analyse : à chaque croisement correspond un "mini-cas" envisageant l'une des technologies sous un angle d'analyse particulier. Chaque mini-cas est donc à la fois contextualisé dans le cas qui lui est propre et relié à d'autre mini-cas relevant du même angle d'analyse. Des " combinaisons thématiques " intégrant différents mini-cas (et proposant donc des parcours de lectures particuliers) propres à un axe d'analyse commun sont en outre proposées à l'utilisateur. 
apprentissage. Au lieu de tout mettre en œuvre pour que l'utilisateur "s'y retrouve" d'emblée et ne soit jamais interrompu dans son activité, il s'agit ici de tirer parti de la difficulté que représente la compréhension de la structure de l'hyperdocument, et de faire en sorte que les efforts déployés pour comprendre cette structure complexe soient exploités dans le cadre de la compréhension du contenu du document.

\section{Conciliation des deux points de vue}

Comment deux thèses aussi différentes que 1'“ utilisabilité " et la CFT peuvent-elles être tenues ? A bien y regarder on peut en fait les articuler.

Pour de nombreux auteurs (Hutchins, Bodker, Norman, Hollan ${ }^{1}$ ), toute médiation d'artefact entre une personne et un objet ou un environnement introduit initialement un gouffre entre nos tentatives d'action-évaluation et le monde. Ce gouffre peut être comblé, soit par une design adapté de l'interface de l'artefact, soit en passant par un travail d'apprentissage de l'utilisateur. Si ce gouffre est suffisamment comblé, l'individu exécute les opérations sur l'artefact sans en être véritablement conscient, considérant d'une certaine manière qu'il opère directement sur l'objet final. Ce comportement automatique permet de focaliser l'attention sur un domaine d'action tout en exécutant facilement les opérations requises dans un autre domaine (une dactylo tape un texte de manière automatique tout en se concentrant sur la construction des phrases suivantes).

Le concept flux d'activité ${ }^{2}$ décrit précisément un cycle d'action dans lequel la tâche, l'artefact et l'objet (ou l'environnement) entretiennent une relation si cohésive qu'elle donne une sensation d'engagement direct. Les interruptions et les résultats inattendus perturbent le flux d'activité et obligent à un effort conscient d'attention sur la tâche.

1 Cf. notamment E. Hutchins, J.Hollan et D. Norman, “ Direct Manipulation Interfaces ", in D. NORMAN et S. W. DRAPER (dir.), User Centered System Design: New Perpectives in Human-Computer Interaction, Hillsdale, NJ, Lawrence Erlbaum Associate, pp. 339-352.

2 Cf. S. BodKer, "A human Activity Approach to User Interfaces", HumanComputer Interaction, vol. 4, pp. 171-195. 
Dans de nombreuses circonstances, cette perturbation du flux d'activité qui porterait à la conscience l'activité perturbée, peut avoir un effet négatif sur la performance. L'activité perturbatrice interfère avec le maintien de la mémoire active sur la tâche qui devrait être au centre de l'attention.

Mais dans d'autres circonstances, l'interruption délibérée du flux d'activité peut avoir un effet positif. Elle peut avoir pour fonction d'obliger l'attention consciente à se tourner sur des aspects importants de la tâche. C'est un procédé fréquemment utilisé dans les situations où 1 'artefact doit prendre en compte le risque lié aux conséquences de certaines opérations exécutées durant la tâche (de la check-list utilisée dans la cabine de pilotage d'un avion aux pop-ups d'avertissement dans les systèmes d'exploitation informatiques). Norman ${ }^{1}$ nomme les éléments du dispositif qui remplissent ce rôle d'interruption délibérée du flux normal d'activité les "fonctions contraignantes" (forcing function).

Reprenons ce qui vient d'être dit dans le cadre de la navigation hypertextuelle. Celle-ci, nous l'avons dit, constitue un objet de mobilisation cognitive particulier pour l'utilisateur. Les partisans de l'usability font tout pour réduire le poids cognitif propre à la navigation et à l'appréhension de la structure du document consulté, considérant que -dans la mesure du possible- le flux d'activité de l'utilisateur, ne doit pas être interrompu. L'utilisateur doit pouvoir rester centré sur son propre projet de consultation (et plus spécialement sur les tâches informationnelles relatives à cette consultation).

Le point de vue alternatif présenté plus haut, à partir des thèses de la CFT, revient à exploiter cette difficulté comme un avantage dans un cadre pédagogique. Il s'agit alors d'envisager les potentialités des "fonctions contraignantes" d'une interface de navigation hypertextuelle, forçant l'utilisateur à s'interroger sur son parcours de navigation.

Le concepteur, au travers de l'interface, joue alors la carte de l'interruption volontaire du flux d'activité, et fait irruption dans le projet de consultation de l'utilisateur pour l'articuler avec un projet (éducatif) qui lui est propre. Ce n'est qu'en suscitant un retour réflexif de la part de l'utilisateur sur sa propre activité de navigation et sur la

1 D. Norman, “ Cognitive Artifacts” in J. M. CARoll (dir.), Designing Interaction : Psychology at the Human-Computer Interface, Cambridge, Cambridge University Press, 1991. 
complexité de la structure qu'il navigue que le concepteur peut espérer initier l'utilisateur à la complexité des savoirs qu'il entend diffuser.

\section{Fonctionnel - rationnel, usability - flexibility, quelles zones de recouvrement?}

Les lignes qui précèdent suggèrent incidemment certaines proximités entre les pôles des deux axes de structuration détaillés : fonctionnel ou rationnel, centré sur l'utilisateur ou centré sur la complexité des savoirs diffusés. Tentons de clarifier les zones de chevauchement possibles entre ces deux axes.

Historiquement, le concept d'usability et le user-centered design se sont développés à partir d'artéfacts hautement intransitifs : cabines de pilotage d'avion, centres de contrôle de centrales nucléaires, outils de réservation de vols aérien, etc. A chaque fois, il s'agit de dispositifs conçus pour réaliser une tâche délimitée et ciblée. Même dans son ouvrage consacré au design des objets quotidiens, Norman ${ }^{1}$ se centre sur des objets ayant une fonction circonscrite : portes, téléphones, cuisinières, chaudières, caméras, etc. Une telle approche devrait donc favoriser la structuration fonctionnelle, se calquant sur la structure de la tâche à mener à bien.

Par ailleurs, l'ambition exprimée par les tenants de la CFT est de susciter l'appropriation de connaissances hautement transitives, car applicables dans une multiplicité de contextes. Dans un tel cadre, la structuration rationnelle semblerait donc plus appropriée.

N'en concluons pas cependant que ces associations sont stables et définitives. En effet, si l'usability impose au concepteur de se conformer aux besoins de l'utilisateur, la façon la plus "extrême " de prendre ces besoins en compte est d'adopter une structuration fonctionnelle, c'est-à-dire de standardiser (au risque de cadenasser) la tâche à réaliser et du même coup le flux d'activité. Mais il est aussi envisageable de prendre le parti inverse, et d'adopter une structuration rationnelle afin d'autonomiser au maximum l'utilisateur, en lui fournissant un document structuré de façon logique, dans lequel il ira "puiser" ça et là les informations dont il a besoin.

1 D. NoRman, The Design of Everyday Things, op cit. 
D'autre part, si la CFT vise l'acquisition de connaissances transitives, les documents conçus selon ses préceptes n'en présentent pas pour autant des informations abstraites de tout contexte d'exploitation. Plutôt, le parti pris par les tenants de la CFT est de présenter l'information dans des contextes multiples et variés. Le point de vue adopté est en quelque sorte "méta-fontionnel ", et non rationnel en tant que tel.

\section{Conclusions}

L'engouement suscité par l'hypertexte tient essentiellement à son caractère réticulaire. Cette potentialité technique qui permet de faire des associations entre des fragments d'informations sur un mode plus libre que ne le permettent les supports classiques a certes comme avantage de pouvoir être plus en conformité avec la structure représentationelle de certains concepts ou théories, qui souffrent de devoir être explicités linéairement, avec un début et une fin, alors qu'ils sont profondément de nature circulairel.

On a vu que cette conformité structurale n'en fait pas pour autant le vecteur privilégié pour l'apprentissage de tels concepts, dans la mesure où le processus de construction des connaissances de l'apprenant peut avoir besoin de passer par des structurations intermédiaires de ces connaissances -structurations sans doute plus sommaires que la structure finalisée du savoir de l'expert.

D'autre part, on a également vu que, si l'hypertexte présente un nouveau mode d'organisation de l'information, malgré tout, les formes structurelles qu'il engendre ne se donnent jamais directement à voir. L'hypertexte montre des items, jamais des liens. Il propose une expérience immersive qui ne permet que des saisies locales de son sujet. C'est à travers la navigation seulement, dans le passage d'un hyperlien à l'autre, que l'utilisateur peut éprouver certains effets de contiguïté ou de proximité -encore peu définis- entre différents fragments d'information.

Donc, en dépit de ses qualités structurantes importantes, l'hypertexte n'est pas de lui-même en mesure de les rendre directement

1 Pensons notamment aux concepts issus de la cybernétique ou des approches systémiques, impliquant des processus de feedback ou de rétroaction, qui se prêtent mal à la mise à plat linéaire propre au texte traditionnel. 
manifeste à celui qui le consulte. Le passage à ce méta niveau qui permettrait une appréhension globale de la configuration d'une matière ou d'un sujet n'est réalisable que, (1) soit à partir d'un important travail interprétatif, inférenciel de l'utilisateur, - (2) soit à l'aide du concours d'autres auxiliaires sémiotiques: les interfaces de navigation.

Mais dans le second cas, il faut garder à l'esprit que le travail de ces interfaces obéit lui aussi à certaines contraintes sémiotiques qui ont un impact sur les contenus communiqués. En effet, la présentatification des liens qui organisent l'hypertexte, réalisée par l'interface, doit, pour être mené à bien, procéder par une explicitation de nature formalisante.

Certes, il est partiellement possible d'exprimer une notion de relation de façon informelle, comme l'ont par exemple brillamment montré les travaux sur la graphique de Jacques Bertin. Mais de tels procédés graphiques ont des difficultés à exprimer plus que des degrés de proximité entre différentes informations. La nature de ces rapports de proximités (qui peuvent être multiples) est, elle difficilement exprimable de manière informelle. Au fond, on retrouve ici le vieux problème, bien connu, des représentations analogiques, incapables de figurer les fonctions logiques. De nombreux auteurs, de Bateson ${ }^{1}$ à Gauthier ${ }^{2}$, ont analysé cette question, montrant la faiblesse de telles représentations à signifier d'autres relations logiques que la conjonction -la négation leur est interdite- et la difficulté pour ces représentations à même figurer la causalité à travers ces relations conjonctives. La saisie des structures globales des hypertextes ne pourra donc pas faire l'économie d'un travail de formalisation symbolique qui expose les articulations hypertextuelles.

De telles particularités ne sont pas sans conséquence dans le champ de la transmission des savoirs.

a. L'hypertexte, seul, ne se révèle pas adapté à une appréhension commode ou facilitatrice de configuration globale de savoirs. Il se révèle approprié, dans des situations où il est attendu de la part de l'utilisateur un effort particulièrement important d'interprétation et de reconstruction.

1 G. BATESon, Vers une écologie de l'esprit (trad. Férial Drosso), Paris, Éd. du Seuil, 1977-1980.

2 G. GAUTHIER, 20 leçons sur l'image et le sens, Paris, Edilig, 1982. 
$b$. Accompagné d'une interface $a d-h o c$, il est susceptible de répondre malgré tout à ces attentes mais sous un mode plutôt formel. Si l'on espère pouvoir exploiter les potentialités sémio-techniques de l'hypertexte dans le but de mettre intuitivement en évidence des configurations articulées de micro-savoirs, comme semblent l'espérer certains acteurs du monde de la pédagogie ou de la communication socio-éducative, on fait fausse route. Une telle mise en évidence passe par un travail inévitable de thématisation des structures, qui est de nature formelle.

Pour reprendre le constat qui ouvrait cet article, expliciter la structure d'un hypermédia, c'est en préciser la forme. Qui dit précision accrue de la forme dit formalisation; la boucle est donc bouclée. 